\title{
Commensal cocktail suppresses gut inflammation
}

The sometimes remarkable therapeutic benefits of fecal transplantation-the transfer of stool from a healthy individual to a diseased one-in patients with severe Clostridium difficile infection illustrate the ability of commensal bacteria to counter dysfunctional human intestinal physiology. As fecal transplantation remains, however, a somewhat invasive and unpalatable procedure used in only the most severe cases, efforts are underway to develop defined mixtures of human microbiota capable of more subtly tuning particular mediators of gut inflammation. In a paper recently published in Nature ${ }^{1}$, Kenya Honda and colleagues report doing just that.

The mediator of interest is the regulatory $T$ $\left(T_{\text {reg }}\right)$ cell population, which suppresses inflammation and is depleted in germ-free mice that lack commensal bacteria. Having previously observed a boost in the number of $T_{\text {reg }}$ cells in the colon of germ-free mice after these mice ingested chloroform-resistant, spore-forming bacteria from normal mouse feces, the team searched for similar $T_{\text {reg-boosting bacteria in }}$ the human gut microbiota ${ }^{2}$. Sure enough, ingestion of the chloroform-resistant fraction of human feces induced the appearance of $T_{\text {reg }}$ cells in the colon of germ-free mice. To identify the particular bacterial strains driving $T_{\text {reg }}$ development, Honda and co-workers cloned over 400 bacterial colonies from the guts of these mice. Genomic analysis revealed that all clones were members of the genus Clostridium and none encoded strong virulence-related genes. A mixture of only 17 of these clostridia strains was sufficient to recapitulate the $T_{\text {reg }}$ cell-promoting effect of the entire chloroformresistant feces fraction and to reduce the severity of experimentally induced allergic diarrhea and colitis. This effect was robust, as it was observed in mice of different genetic backgrounds and in rats.

Fredrik Bäckhed at the University of Gothenburg, Sweden, concludes that "this is a really important study because the field is a little bit stuck, in that most mechanistic studies are done in mice, and most correlative studies are done in humans. This particular study is a step forward because it represents an intermediate between mouse and man."

This study might also be considered surprising in light of a previous report that mouse, but not human, fecal microbiota induced maturation and expansion of T cells in the intestines of germ-free mice ${ }^{3}$. However, a closer look reveals that the different overall conclusions of these two studies are likely related to their

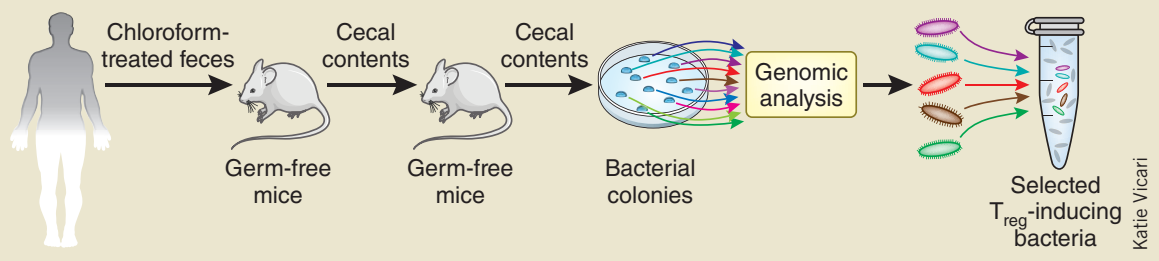

focus on different anatomical sites. More specifically, whereas human microbiota did not seem to reconstitute $T$ cells in the small intestine, both studies detected robust effects of human microbiota on T cells in the colon. The mechanisms explaining this potential anatomical effect remain unclear.

In terms of mechanism, the 17 strains studied by Honda and colleagues clearly act synergistically because no single strain, or even mixtures of 3 or 5 of the strains, had the same

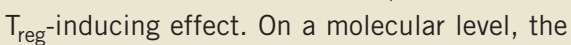
17-strain cocktail seems to exert several effects on $T_{\text {reg }}$ cell differentiation. First, short-chain fatty acids in the cecal contents of colonized mice elicit production of transforming growth factor $\beta$ - a known inducer of $T_{\text {reg }}$ cell differentiation and expansion-from intestinal epithelial cells. Second, bacterial antigens in the cocktail seem capable of directly stimulating $T_{\text {reg }}$ cell suppressive activity, at least in vitro. Even so, further work is needed to measure the relative contributions of these and other mechanisms to the $T_{\text {reg-potentiating effect of this probiotic }}$ cocktail. "I have no doubt that multiple mechanisms are at play. I think it's safe to say that the most important molecules involved in this activity have not yet been discovered," says Michael Fischbach from the University of California, San Francisco.

In fact, if defined molecular 'active principles' accounting for the $T_{\text {reg-potentiating effect of the }}$ 17-strain mixture are identified, it is possible that administration of the purified molecules, rather than the bacteria themselves (by means of an enema, for example), would be sufficient to boost $T_{\text {reg }}$ cell numbers. Also unclear is whether this bacterial cocktail will be able to exert clinically meaningful therapeutic effects if administered in a scenario of ongoing severe intestinal inflammation. This is because, although Honda and colleagues demonstrated beneficial effects on experimental allergic diarrhea and colitis, the probiotic was administered just before or at the same time as disease onset, in a more prophylactic than therapeutic regimen. Although confirming that "the principle that a therapy like this will be able to turn things around, in other words induce $T_{\text {reg }}$ differentiation in the face of ongoing inflammation, has not been demonstrated yet," Fischbach states that "the field is very optimistic that this will be possible." Another reason for optimism is that mining of publicly available human microbiome data revealed that several of the strains in the 17-strain cocktail were depleted in the microbiomes of ulcerative colitis patients compared with healthy humans.

Yet another question is what sorts of studies will be needed to bring a probiotic like this to the clinical market. This 17-strain mixture differs from many existing probiotics in several ways. For one, it consists of actual human commensal microbes, rather than noncommensal strains, selected in part because they are easy to culture or package. For another, it contains multiple rather than a single bacterial strain. Both of these features may influence its efficacy in humans and its chances of gaining regulatory approval. Bäckhed explains that "because there are big variations in the composition of the microbiome in different individuals, a probiotic composed of just one lactobacillus strain may be effective in some people but not all. A cocktail of several bacteria has a larger likelihood of effectively treating a larger portion of the human population. Also, if all of the strains in the cocktail are commensal bacterial strains known not to be harmful, including five strains instead of one strain will not likely markedly increase risk."

Nevertheless, by sequencing the genomes of all bacterial strains in the 17-strain cocktail, Honda and colleagues may already be one step ahead of the regulatory process. According to Fischbach, "For any therapeutic products that will be mixtures of purified bacterial strains, the genome sequences will need to be known, at the very least to rule out the presence of cryptic toxins. The question is how much additional characterization of these strains will be required to bring something safe to the clinic."

Christine Borowski, Senior Editor, Nature Biotechnology

1. Atarashi, K. et al. Nature doi:10.1038/nature12331 (10 July 2013).

2. Atarashi, K. et al. Science 331, 337-341 (2011).

3. Chung, H. et al. Cell 149, 1578-1591 (2012). 